\title{
Comparison of Quantiferon Test with Tuberculin Skin Test for the Detection of Tuberculosis Infection in Children
}

\author{
Hatice Onur, ${ }^{1}$ Sami Hatipoğlu, ${ }^{2}$ Vefik Arıca, ${ }^{3,6}$ Nevin Hatipoğlu, ${ }^{4}$ and Seçil Gunher Arıca ${ }^{5}$
}

\begin{abstract}
The efficacy of Quantiferon-TB gold test (QFT-GIT) remains to be documented in pediatric population. Tuberculin skin test (TST) is a conventional test available for the diagnosis of latent tuberculosis infection (LTBI). We aimed to investigate the concordance between QFT-GIT and TST in children with and without tuberculosis infection. Ninety-seven patients, aged 3 months14 years, admitted to pediatric outpatient clinics of Dr. Sadi Konuk Training Hospital Bakırköy, Turkey between March 2008 and April 2009 were recruited. Demographic features, TST results, history of exposure to active tuberculosis (TB), chest X-ray findings, clinical history, presence of Bacillus Calmette Guerin (BCG) vaccination scar were recorded. Patients were categorized into four groups namely, active TB, LTBI, no TB and healthy. It was found that BCG scar positivity did not influence QFT-GIT results. There was a statistically significant agreement between QFT-GIT and TST results $(\kappa=0.486 ; p<0.01)$. In patients $\geq 5$ years of age, TST positivity and QFT positivity had a significant relationship $(p<0.01)$. In all patient groups, sensitivity and specificity was $65.85 \%$ and $82.14 \%$, respectively. In active TB group, TST and QFT-GIT results demonstrated significant agreement ratio of $40.8 \%(\kappa=0.364 ; p<0.01)$. Sensitivity and specificity was $100 \%$ and $30 \%$, respectively. Utilization of QFT-GIT in the diagnosis of LTBI reduces false-positive results and prevents unnecessary treatment with INH and its adverse effects.
\end{abstract}

KEY WORDS: tuberculosis; quantiferon; tuberculin skin test; BCG.

\section{INTRODUCTION}

Number of cases with tuberculosis infection increased at the beginning of 21 st century. It is reported that one third of world's population is infected with Mycobacterium tuberculosis and 1.7 million deaths worldwide are due to active tuberculosis. It is documented that over 1 million new cases of tuberculosis is diagnosed in children younger than 15 years of age annually and active disease accounts

\footnotetext{
$\overline{{ }^{1} \text { Department }}$ of Pediatric, İstanbul Training and Research Hospital, İstanbul, Turkey

${ }^{2}$ The Chair of Pediatric Clinic, Dr Sadi Konuk Training and Research Hospital, İstanbul, Turkey

${ }^{3}$ Department of Pediatric, Mustafa Kemal University Medical Faculty, Serinyol, 31100 Antakya, Hatay, Turkey

${ }^{4}$ Department of Infectious Diseases, Kanuni Sultan Süleyman Training and Research Hospital, İstanbul, Turkey

${ }^{5}$ Department of Family Medicine, Mustafa Kemal University Medical Faculty, Antakya, Hatay, Turkey

${ }^{6}$ To whom correspondence should be addressed at Department of Pediatric, Mustafa Kemal University Medical Faculty, Serinyol, 31100 Antakya, Hatay, Turkey. E-mail: vefikarica@hotmail.com
}

for 400,000 deaths in this population [1]. In Turkey, annual number of new TB cases reported to World Health Organization (WHO) is approximately 20.000; and the incidence of TB is 30/100,000 [1].

TB is diagnosed by combining patient's history of exposure to active disease, tuberculin skin test (TST) results, clinical and radiological findings. Microbiologic tests yield positive results in only $30-40 \%$ of cases [2]. TST poses some technical problems such as the potential for false-positive and false negative results and problems in administration and interpretation [2].

Identification of Early Secreted Antigenic Target$6 \mathrm{kD}$ protein (ESAT-6) and Culture Filtrate Protein-10 $\mathrm{kD}$ (CFT-10) has been a promising development in the diagnosis of TB. These antigenic proteins are encoded within the region of difference 1 (RD 1) of the $M$. tuberculosis genome. Neither of these proteins exist in Bacillus Calmette Guerin (BCG) and non-tuberculosis mycobacteria making them more specific tools in the diagnosis of M. tuberculosis [3]. Quantiferon-TB gold test (QFT-GIT), licensed by FDA in 2004, utilizes ESAT-6, 
CFT-10 and TB7.7 antigens for stimulating in vitro release of $\gamma$-IFN from memory T cells [4]. Released $\gamma$-IFN level is measured by enzyme-linked immunosorbent assay (ELISA) method. Majority of the research about $\gamma$-IFN was conducted in countries of low TB incidence and mainly in adults. However, in countries such as Turkey where TB is endemic, more studies are required to document the efficacy of QFT-GIT in pediatric population.

We aimed to investigate the agreement between QFT-GIT and TST results in children with and without tuberculosis infection.

\section{MATERIALS AND METHODS}

Ninety-seven patients admitted to pediatric outpatient clinics of Dr. Sadi Konuk Training Hospital in Bakırköy, Turkey between March 2008 and April 2009 were enrolled. The age of the patients ranged from 3 months to 14 years. Demographic features, TST results, history of exposure to active tuberculosis (TB), chest X-ray findings, clinical history, presence of BCG vaccination scar were recorded. Patients were categorized into four main groups namely, active $\mathrm{TB}$, latent tuberculosis infection (LTBI), no TB, and healthy.

Group I $(n=30)$ : Active TB; patients with signs and symptoms suggestive of $\mathrm{TB}$ and diagnostic work-up yielded the diagnosis of $\mathrm{TB}$, finally anti-TB chemotherapy started.

Group II $(n=15)$ : LTBI, patients were asymptomatic but TST results were positive.

Group III $(n=27)$ : Non-TB, patients with signs and symptoms suggestive of TB however diagnostic work-up excluded the disease.

Group IV $(n=25)$ : Healthy children.

Written parental informed consent was obtained for each patient. The study was approved by the hospital ethics committee.

Exclusion Criteria Patients with hemodynamically unstable cardiopulmonary disease, history of severe allergic reaction to purified protein derivatives (PPD), history of active TB, immunodeficiency, malnutrition and patients whose parent/ guardian refused to consent were excluded.

TST Administration Tuberculin skin test was performed on the volar aspect of the forearm by administering $0.1 \mathrm{ml}$ (5 tuberculin units) of PPD solution intradermally. Vertical and horizontal diameter of the induration was measured $48-72 \mathrm{~h}$ after the injection by using the ball point pen method.

TST result $\geq 10 \mathrm{~mm}$ was considered positive in patients without a BCG vaccination scar and TST result $\geq 15 \mathrm{~mm}$ was considered positive in patients with $\mathrm{BCG}$ vaccination scar. In patients with $B C G$ vaccination scars, TST $<15 \mathrm{~mm}$ was considered negative [5].

\section{QFT}

The QFT assay was performed as per the manufacturer's instructions. The assay involved two stages: the first stage involved incubation of whole blood with antigens, and the second stage involved measurement of IFN-g production in harvested plasma by ELISA. Venous blood was directly collected, prior to TST administration, into three 1-ml heparin-containing tubes. One tube contained only heparin as negative control, another also contained mitogen as positive control, and the third tube had overlapping peptides representing the entire sequences of ESAT- 6 and CFP-10 and another peptide from a portion of the TB antigen TB7.7 (Rv2654). Within 2-6 h of blood draw, the tubes were incubated at $37^{\circ} \mathrm{C}$. After exactly $24 \mathrm{~h}$ of incubation, the tubes were centrifuged and plasma was harvested and frozen at $-70^{\circ} \mathrm{C}$ until the ELISA was performed (on average, ELISA was performed within 46 weeks of blood collection). The $\gamma$-INF response was quantified using ELISA (Cellestis Ltd, Carnegie, Victoria, Australia). $\gamma$-INF values (in international units per milliliter) for TB-specific antigens and mitogen were corrected for back ground by subtracting the value obtained for the respective negative control. As recommended by the manufacturer, and based on previous studies, the cut-off value for a positive test was $\gamma-\mathrm{INF} \geq 0.35 \mathrm{IU} / \mathrm{ml}[6,7]$.

Statistical Analysis NCSS 2007 and PASS 2008 Statistical Software (Utah, USA) program was used for statistical analysis. Because there is no gold standard for diagnosis of LTBI, concordance between TST and QFT-GIT was assessed by using proportion agreement and kappa coefficients. Chi-square and Fisher's exact tests were used for categorical variables. A $p$ value of less than 0.05 was considered significant and results were reported with $95 \%$ confidence intervals.

\section{RESULTS}

A total of 97 patients were enrolled. Forty eight (49.5\%) patients were female. Age and gender of 
Table 1. Demographic and Clinical Features of Patients

\begin{tabular}{|c|c|c|c|c|c|c|}
\hline$N$ & $\begin{array}{l}\text { Active TB } \\
30\end{array}$ & $\begin{array}{l}\text { LTBI } \\
15\end{array}$ & $\begin{array}{l}\text { Healthy } \\
25\end{array}$ & $\begin{array}{l}\text { Non-TB } \\
27\end{array}$ & $\begin{array}{l}\text { Total } \\
97\end{array}$ & $\chi^{2} ; p$ \\
\hline \multicolumn{7}{|l|}{ Gender, $n(\%)$} \\
\hline Female & $15(50 \%)$ & $8(53.3 \%)$ & $10(40 \%)$ & $15(55.6 \%)$ & $48(49.5 \%)$ & \multirow[t]{2}{*}{$\chi^{2}=1.39 ; p=0.708$} \\
\hline Male & $15(50 \%)$ & $7(46.7 \%)$ & $15(60 \%)$ & $12(44.4 \%)$ & $49(50.5 \%)$ & \\
\hline \multicolumn{7}{|l|}{ Age, $n(\%)$} \\
\hline$<5$ & $9(30 \%)$ & $2(13.3 \%)$ & $4(16 \%)$ & $8(29.6 \%)$ & $23(23.7 \%)$ & \multirow{2}{*}{$\chi^{2}=2.89 ; p=0.408$} \\
\hline$\geq 5$ & $21(70 \%)$ & $13(86.7 \%)$ & $21(84 \%)$ & $19(70.4 \%)$ & $74(76.3 \%)$ & \\
\hline \multicolumn{7}{|l|}{ QFT, $n(\%)$} \\
\hline Positive & $27(90 \%)$ & $10(66.7 \%)$ & $0(0 \%)$ & $0(0 \%)$ & $37(38.1 \%)$ & \multirow[t]{3}{*}{$\chi^{2}=73.08 ; p=0.001 * *$} \\
\hline Negative & $3(10 \%)$ & $5(33.3 \%)$ & $23(92 \%)$ & $23(85.2 \%)$ & $54(55.7 \%)$ & \\
\hline Indeterminate & - & - & $2(8 \%)$ & $4(14.8 \%)$ & $(6.2 \%)$ & \\
\hline \multicolumn{7}{|l|}{$\mathrm{TST}, n(\%)$} \\
\hline Positive & $20(66.7 \%)$ & $12(80 \%)$ & $0(0 \%)$ & $9(33.3 \%)$ & $41(42.3 \%)$ & \multirow{2}{*}{$\chi^{2}=35.25 ; p=0.001^{* *}$} \\
\hline Negative & $10(33.3 \%)$ & $3(20 \%)$ & $25(100 \%)$ & $18(66.7 \%)$ & $56(57.7 \%)$ & \\
\hline \multicolumn{7}{|c|}{ BCG vaccination scar, $n(\%)$} \\
\hline Positive & $24(80 \%)$ & $14(93.3 \%)$ & $23(92 \%)$ & $24(88.9 \%)$ & $85(87.6 \%)$ & \multirow[t]{2}{*}{$\chi^{2}=2.54 ; p=0.468$} \\
\hline Negative & $6(20 \%)$ & $1(6.7 \%)$ & $2(8 \%)$ & $3(11.1 \%)$ & $12(12.4 \%)$ & \\
\hline \multicolumn{7}{|c|}{ History of exposure, $n(\%)$} \\
\hline Positive & $18(60 \%)$ & $9(60 \%)$ & $0(0 \%)$ & $10(37 \%)$ & $37(38.1 \%)$ & \multirow[t]{2}{*}{$\chi^{2}=24.54 ; p=0.001 * *$} \\
\hline Negative & $12(40 \%)$ & $6(40 \%)$ & $25(100 \%)$ & $17(63 \%)$ & $60(61.9 \%)$ & \\
\hline \multicolumn{7}{|c|}{ Chest X-ray, $n(\%)$} \\
\hline Positive & $25(83.3 \%)$ & $0(0 \%)$ & - & $16(59.3 \%)$ & $41(56.9 \%)$ & \multirow[t]{2}{*}{$\chi^{2}=28.42 ; p=0.001^{* *}$} \\
\hline Negative & $5(16.7 \%)$ & $15(100 \%)$ & - & $11(40.7 \%)$ & $31(43.1 \%)$ & \\
\hline
\end{tabular}

the patients did not differ significantly $(p>0.05$; Table 1).

Number of positive QFT results differed significantly between groups $(p<0.01)$ QFT positivity rate was significantly higher in group I and II compared to other groups. Rate of indeterminate test results was significantly higher in group III compared to other groups (Fig. 1, Table 1). TST positivity was different between groups $(p<0.01)$. The number of positive TST results was significantly lower in group IV compared to other groups (Fig. 2, Table 1). Numbers of BCG vaccination scar-positive children were close in study groups $(p>0.05$; Table 1$)$. History of exposure to active TB was significantly different between all groups $(p<0.01)$, it was especially low in group IV (Table 1). Chest X-ray abnormalities were observed in $83.3 \%, 0 \%$, and $59.3 \%$ of cases with active TB, LTBI, and non-TB, respectively ( $p<0.01$; Table 1$)$.

In all cases and groups, presence of BCG vaccination scar influenced neither TST nor QFT results $(p>0.05)$. QFT results were not affected by TST induration diameters; rate of QFT positivity was significantly higher in patients with an induration diameter of $10-14$ and $\geq 15 \mathrm{~mm}$ compared to other diameters (Table 2).
In active disease group; QFT results were positive in all patients with positive TST results. In LTBI group, TST positivity was not associated with QFT positivity $(p>0.05)$. In non-TB group, there was not a significant relationship between TST positivity and QFT positivity $(p>0.05)$. When all cases were considered; there was a significant relationship between TST positivity and QFT positivity $(p<0.01)$; TST positivity rate was significantly higher in patients with positive QFT results (Table 3 ).

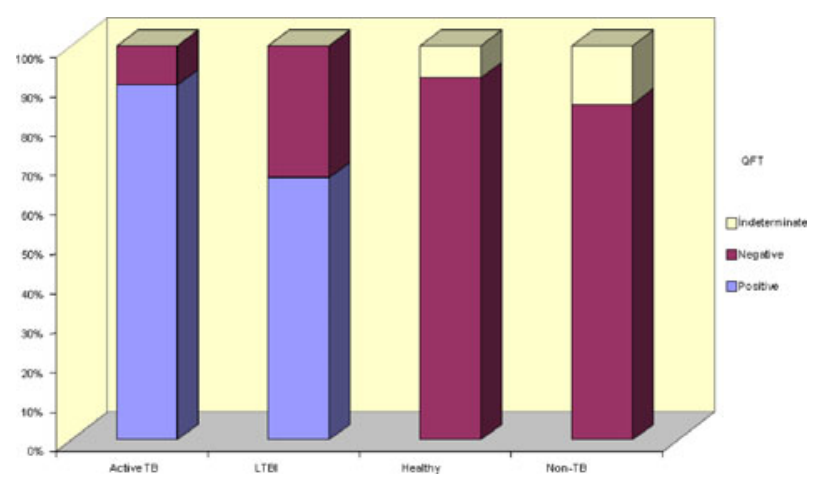

Fig. 1. Distribution of QFT results for all groups of patients. 


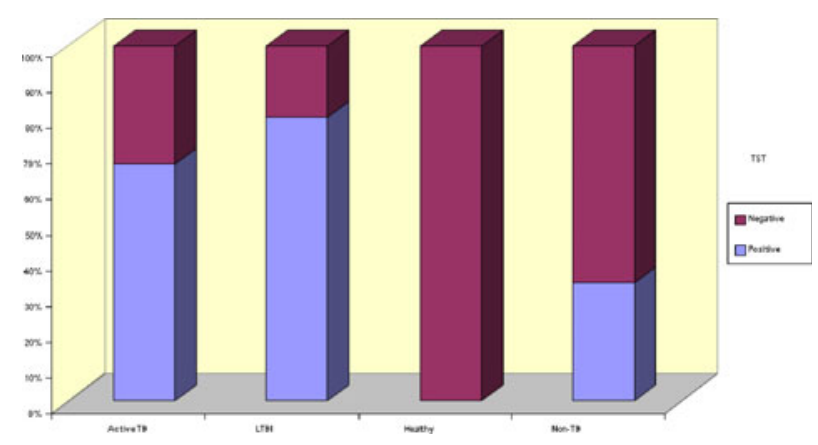

Fig. 2. Distribution of TST results for all groups of patients.

In active disease group, non-incidental agreement rate between TST and QFT was $40.8 \%(\kappa=0.364 ; p<0.01)$. In this group, sensitivity (i.e., rate of positive QFT results in patients with positive TST results) was $100 \%$ and specificity (i.e., rate of negative QFT results in patients with negative TST results) was $30 \%$. Positive predictive value was $74.07 \%$; negative predictive value was, accuracy of the test was $76.7 \%$ (Table 3).

In LTBI group, non-incidental agreement rate between TST and QFT was $-33.3 \%(\kappa=-0.333 p>0.05)$. In this group sensitivity was $58.33 \%$ and specificity was $0 \%$. Positive predictive value was $70 \%$; negative predictive value was $0 \%$, the accuracy of the test was $46.67 \%$ (Table 3).

In all cases, non-incidental agreement rate between TST and QFT was $48.6 \%(\kappa=0.486 ; p<0.01)$. In this group, sensitivity was $65.85 \%$ and specificity was $82.14 \%$. Positive predictive value was $72.97 \%$; negative predictive value was $76.67 \%$, the accuracy of the test was $75.26 \%$ (Table 3 ).

In patients $<5$ years of age, there was not a significant relationship between TST positivity and QFT positivity $(p>0.05)$. Non-incidental agreement rate between TST and QFT was $25.1 \%(\kappa=0.251 ; p>0.05)$.
In this group, sensitivity was $42.85 \%$; specificity was $92.5 \%$. Positive predictive value was $50 \%$; negative predictive value was $90.24 \%$; the accuracy of the test was $85.1 \%$ (Table 4 ).

In patients $\geq 5$ years of age; there was a significant relationship between TST positivity and QFT positivity $(p<0.01)$; positive TST result rates were significantly higher in patients with positive QFT results. Nonincidental agreement rate between TST and QFT was $53.4 \%(\kappa=0.534 ; p<0.01)$. In this group, sensitivity was $70.58 \%$; specificity was $82.50 \%$. Positive predictive value was $77.41 \%$; negative predictive value was $74.74 \%$ and the accuracy of the test was $77.03 \%$ (Table 4)

\section{DISCUSSION}

Recently emerged tests, based on measuring in vitro release of IFN- $\gamma$ from T- lymphocytes upon exposure to mycobacterial antigens, are considered as promising alternative tools to TST in the diagnosis of TB.

Several authors have emphasized that BCG vaccination leads to false positive TST results and they reported that specificity of IFN- $\gamma$ release assays (IGRA) might be higher [8-10]. TST induration diameter is influenced by the age at which the first BCG dose is received, interval between two doses, and amount of the administered vaccine dose [11, 12]. It is reported that in developed countries, BCG vaccination at birth or during infancy does not affect TST results [7]; however, BCG vaccination affects the sensitivity of TST in underdeveloped and developing countries $[1,13]$.

It is clearly documented that BCG vaccination does not affect QFT test results [14-18]. Our results parallels the results of previous research and indicates that $\mathrm{BCG}$

Table 2. Agreement Between TST and QFT Results

\begin{tabular}{|c|c|c|c|c|c|c|}
\hline \multirow[b]{2}{*}{ QFT } & \multicolumn{5}{|c|}{ Diameter of induration (mm) } & \multirow[b]{2}{*}{$p$} \\
\hline & $\begin{array}{l}0-5 \\
n(\%)\end{array}$ & $\begin{array}{l}5-9 \\
n(\%)\end{array}$ & $\begin{array}{l}10-14 \\
n(\%)\end{array}$ & $\begin{array}{l}\geq 15 \\
n(\%)\end{array}$ & $\begin{array}{l}\text { Total } \\
n(\%)\end{array}$ & \\
\hline Positive & $4(12.5 \%)$ & $0(0 \%)$ & $7(50 \%)$ & $26(65 \%)$ & $37(38.1 \%)$ & $0.001 * *$ \\
\hline Negative & $26(81.3 \%)$ & $10(90.9 \%)$ & $6(42.9 \%)$ & $12(30 \%)$ & $54(55.7 \%)$ & \\
\hline Indeterminate & $2(6.3 \%)$ & $1(9.1 \%)$ & $1(7.1 \%)$ & $2(5 \%)$ & $6(6.2 \%)$ & \\
\hline Total & $32(100 \%)$ & $11(100 \%)$ & $14(100 \%)$ & $40(100 \%)$ & $97(100 \%)$ & \\
\hline
\end{tabular}

$* * p<0.01$ 
Table 3. Agreement Between QFT and TST Results

\begin{tabular}{lllccccc}
\hline Variable & $n$ & Kappa value $(p$ value $)$ & Positive agreement $\%$ & Negative agreement $\%$ & TST/-QFT \% & - TCTD/+QFT \% & Accuracy $(\%)$ \\
\hline Active TB & 29 & $\kappa=0.364 ; p=0.006^{* *}$ & 100 & 30.00 & 74.07 & 700 \\
LTBI & 15 & $\kappa=-0.333 ; p=0.171$ & 58.33 & 0 & 70.0 & 0 \\
All cases & 97 & $\kappa=0.486 ; p=0.001^{* *}$ & 65.85 & 82.14 & 76.67 & 72.97 \\
\hline
\end{tabular}

$* * p<0.01$

vaccination status and QFT results do not pose a statistically significant relationship $(p>0.05)$. In populations of widespread BCG vaccine use, QFT may be an alternative tool to TST in the diagnosis of TB.

It is clearly stated in the guideline of Centers for Disease Control and Prevention that since the sensitivities are rather suboptimal, negative QFT and TST results do not rule-out active TB [19]. In various studies comparing TST and QFT, sensitivity was found to be between $55-88 \%$ and mean value of sensitivity was $75 \%$ [20]. Indeterminate results observed in $7 \%$ of patients were attributed to the immunosuppresion due to active TB. It is observed that indeterminant results are detected more frequently in studies encompassing patients with immunodeficiency [20]. In our study, none of the results were indeterminate in patients with active TB. This finding might be attributed to the fact that patients with immunodeficiency were excluded from our study.

In Japan, Kobashi et al. conducted a study which included 50 healthy volunteers, 50 patients with active TB, and 100 patients with non-TB infections and emphasized that QFT is superior to TST in differentiating active TB from non-TB Mycobacterium infections [21].

Higucki et al. compared TST and QFT results of college students who had a history of exposure to an index case with active TB and followed-up these students for 3.5 years. In conclusion, authors recom- mended utilization of QFT instead of TST while investigating children with a history of exposure to active TB and they proposed that positive TST results should be confirmed via QFT [22].

Mazurek et al. published a QFT-G instruction guideline in 2005. In this guide, they reported that QFT-G, similar to TST, was not an effective tool for differentiating LTBI from active TB and stressed the importance of utilizing radiologic examinations, clinical findings, microbiologic, and cytologic investigations for a firmly established diagnosis of TB. The authors reported that neither negative TST results, nor negative QFT results excluded diagnosis of TB infection and added that in the presence of symptoms and signs suggesting diagnosis of TB, negative TST or QFT results might not rule-out TB infection. They also reported the importance of HIV testing in suspicious cases [23]. In our study, non-incidental agreement rate between TST and QFT was $-33.3 \%(\kappa=-0.333 p>0.05)$ in LTBI group; sensitivity was $58.33 \%$ and specificity was $0 \%$. Positive predictive value was $70 \%$; negative predictive value was $0 \%$, the accuracy of the test was $46.67 \%$. TB prevention programs recommend QFT for the follow-up of LTBI in patients with a history of exposure to active disease because QFT makes a second visit unnecessary, is more specific than TST and - contrary to TST-does not have a booster effect [23]. Our findings support this recommendation.

Table 4. Agreement of QFT and TST Results According to Age

\begin{tabular}{|c|c|c|c|c|c|c|}
\hline & & & \multicolumn{3}{|l|}{ TST } & \multirow[b]{2}{*}{$p$} \\
\hline & & & $\begin{array}{l}\text { Positive } \\
n(\%)\end{array}$ & $\begin{array}{l}\text { Negative } \\
n(\%)\end{array}$ & $\begin{array}{l}\text { Total } \\
n(\%)\end{array}$ & \\
\hline \multirow[t]{3}{*}{$<5$ years } & QFT & Positive & $3(42.9 \%)$ & $3(42.9 \%)$ & $6(26.1 \%)$ & 0.318 \\
\hline & & Negative & $4(57.1 \%)$ & $13(81.3 \%)$ & $17(73.9 \%)$ & \\
\hline & & Total & $7(100 \%)$ & $16(100 \%)$ & $30(100 \%)$ & \\
\hline \multirow[t]{3}{*}{$\geq 5$ years } & QFT & Positive & $24(70.6 \%)$ & $7(17.5 \%)$ & $31(41.9 \%)$ & $0.001 * *$ \\
\hline & & Negative & $10(29.4 \%)$ & $33(82.5 \%)$ & $43(57.1 \%)$ & \\
\hline & & Total & $34(100 \%)$ & $40(100 \%)$ & $74(100 \%)$ & \\
\hline
\end{tabular}

Fisher's exact test and Chi-square tests were used ${ }^{*} p<0.05$ 
One of the 26 healthy children had a positive QFT result. Although he did not have any signs and symptoms of infection, chest X-ray, and thorax CT findings revealed active TB and anti-tuberculous chemotherapy was commenced promptly. This patient was assigned as healthy when symptoms, history of exposure, TST result and physical examination findings were evaluated. The patient was diagnosed incidentally by QFT. This finding indicates that QFT, as well as TST, may be used in routine screening of TB in our country. Two of the remaining 25 patients had indeterminate results. Since these patients did not have any clinical features suggestive of TB, repeat QFT was not performed as recommended previously [23].

Connel et al. stated that rate of QFT positivity and mitogen response increased with advancing age [7]. Nakaoka et al. reported a decreased response to QFT in children $<5$ years of age [24]. Ulrich et al. declared that publications indicating a lower response rate to QFT in infants and young children were scarce and more research was needed on that issue [25]. In 2009, Bianchi et al. reported that sensitivity of IGRA was higher compared to TST in children less than 4 years of age [26]. In our study, agreement between QFT and TST was $53.4 \%(\kappa=$ $0.251 ; p>0.05)$ in patients older than 5 years of age and it was $25.1 \%(\kappa=0.534 ; p<0.01)$ in patients younger than 5 years of age. The agreement in patients older than 5 years of age was significant compared to patients younger than 5 years of age. Finally, it can be proposed that QFT may be used routinely for TB monitoring in Turkish children, especially those older than 5 years of age.

It is shown that TST induration diameter and QFT results correlate well $[27,28]$. Johnson et al. failed to document a significant relationship between TST and QFT results [29]. In our study, QFT positivity differed significantly according to TST induration diameter: QFT positivity rate was significantly higher in patients with an induration diameter of $10-14$ and $\geq 15 \mathrm{~mm}$ compared to other diameters $(p=0.001)$. Although QFT-G use is not widespread in our country, children with a TST result between 10 and $14 \mathrm{~mm}$ should be followed-up closely.

In literature, a substantially significant agreement was documented between QFT and TST results [10, 30, 31]. We observed in our study that non-incidental agreement between QFT and TST was $48.6 \%$ and it was statistically significant $(\kappa=0.486 ; p<0.01)$. In this group, sensitivity was $65.85 \%$ and specificity was $82.14 \%$. Positive predictive value was $72.97 \%$; negative predictive value was $76.67 \%$, the accuracy of the test was $75.26 \%$.

To conclude, IFN- $\gamma$ release assays may have numerous advantages over TST such as higher specificity, better relationship with $M$. tuberculosis exposure, and lower cross-reaction rates with BCG vaccination and NTM. Tests based on measurement of released IFN- $\gamma$ may reduce false-positive results and prevent unnecessary treatment with INH and its adverse effects.

Open Access This article is distributed under the terms of the Creative Commons Attribution License which permits any use, distribution, and reproduction in any medium, provided the original author(s) and the source are credited.

\section{REFERENCES}

1. Report WHO 2010, Global tuberculosis control-surveillance, planning, financing. Geneva, Switzerland: 2010.

2. Mandalakas, A.M., A.K. Detjen, A.C. Hesseling, et al. 2011. Interferon-gamma release assays and childhood tuberculosis: systematic review and meta-analysis. The International Journal of Tuberculosis and Lung Disease 15(8): 1018-1032.

3. Barnes, P.F. 2004. Diagnosing latent tuberculosis infection: turning glitter to gold. American Journal of Respiratory and Critical Care Medicine 170: 5-6.

4. Gerberding, J.L. 2005. Guidelines for preventing the transmission of Mycobacterium tuberculosis in health-care settings 54(RR17): 1-141

5. Beyers, N., R.P. Gie, H.S. Schaaf, et al. 1997. A prospective evaluation of children under the age of 5 years living in the same household as adults with recently diagnosed pulmonary tuberculosis. The International Journal of Tuberculosis and Lung Disease 1(1): 38-43.

6. Khan, E.A., and J.R. Strake. 1995. Diagnosis of tuberculosis in children: increased need for better methods. Emerging Infectious Diseases 1: 115-123.

7. Chadha, V.K., P.S. Jagannatha, and P. Kumar. 2004. Can BCG vaccinated children be included in tuberculin surveys to estimate the annual risk of tuberculosis infection in India. The International Journal of Tuberculosis and Lung Disease 8: 1437-1442.

8. Mori, T., M. Sakatani, F. Yamagishi, et al. 2004. Specific detection of tuberculosis infection: an interferon-gamma-based assay using new antigens. American Journal of Respiratory and Critical Care Medicine 170(1): 59-64.

9. Brock, I. 2004. Comparison of tuberculin skin test and new specific blood test in tuberculosis contacts. American Journal of Respiratory and Critical Care Medicine 170: 65-69.

10. Kang, Y.A., H.W. Lee, H.I. Yoon, et al. 2005. Discrepancy between the tuberculin skin test and the whole-blood interferon gamma assay for the diagnosis of latent tuberculosis infection in an intermediate tuberculosis-burden country. Journal of the American Medical Association 293(22): 2756-2761.

11. Rieder, H.L. 1999. Epidemiological basis of TB control, 26-43. Paris: International Union against Tuberculosis and Lung Disease.

12. Wang, L., M.O. Turner, R.K. Elwood, et al. 2002. A meta-analysis of the effect of Bacille Calmette Guérin vaccination on tuberculin skin test measurements. Thorax 57(9): 804-809.

13. Ewer, K., J. Deeks, L. Alvarez, et al. 2003. Comparison of T-cellbased assay with tuberculin skin test for diagnosis of Mycobacte- 
rium tuberculosis infection in a school tuberculosis outbreak. Lancet 361(9364): 1168-1173.

14. Pai, M. 2004. Riley interferon gamma assays in immunodiagnosis of tuberculosis review. Lancet Information 4: 761-776.

15. Pai, M., L.W. Riley, and J.M. Colford Jr. 2004. Interferon-gamma assays in the immunodiagnosis of tuberculosis: a systematic review. The Lancet Infectious Diseases 4(12): 761-776.

16. Diel, R., A. Nienhaus, C. Lange, K. Meywald-Walter, et al. 2006. Tuberculosis contact investigation with a new, specific blood test in a low-incidence population containing a high proportion of BCGvaccinated persons. Respiratory Research 7: 77.

17. Dogra, S., P. Narang, D.K. Mendiratta, et al. 2007. Comparison of a whole blood interferon-gamma assay with tuberculin skin testing for the detection of tuberculosis infection in hospitalized children in rural India. Journal of Infection 54(3): 267-276.

18. Britton, W.J., G.L. Gilbert, J. Wheatley, et al. 2005. Sensitivity of human gamma interferon assay and tuberculin skin testing for detecting infection with Mycobacterium tuberculosis in patients with culture positive tuberculosis. Tuberculosis 85(3): 137-145.

19. Mazurek, G.H., J. Jereb, P. Lobue, et al. 2005. Division of Tuberculosis Elimination, National Center for HIV, STD, and TB Prevention, Centers for Disease Control and Prevention (CDC). Guidelines for using the QuantiFERON-TB Gold test for detecting Mycobacterium tuberculosis infection, United States. MMWR Recommendations and Reports 54(RR-15): 49-55.

20. Pai, M., and D.M. Lewinsohn. 2005. Interferon-gamma assays for tuberculosis: is anergy the Achilles' heel? American Journal of Respiratory and Critical Care Medicine 172(5): 519-521.

21. Kobashi, Y., and Y. Obase. 2006. Clinical reevaluation of the QuantiFERON TB-2G test as a diagnostic method for differentiating active tuberculosis from nontuberculous mycobacteriosis. Clinical Infectious Diseases 43(12): 1540-1546.

22. Higuchi, K., and N. Harada. 2007. Use of quantiferon-TB gold to investigate tuberculosis contacts in a high school. Respirology 12 (1): $88-92$
23. Delacourt, C., J.D. Poveda, and Q. Churean. 1995. Use of polymerase chain reaction for improved diagnosis of tuberculosis in children. Journal of Pediatrics 126: 703-709.

24. Nakaoka, H., and L. Lawson. 2006. Risk for tuberculosis among children. Emerging Infectious Diseases 12(9): 1383-1388.

25. Ulrichs, T., and P. Anding. 2000. Increased numbers of ESAT-6and purified protein derivative-specific gamma interferon-producing cells in subclinical and active tuberculosis infection. Infection and Immunity 68(10): 6073-6076.

26. Bianchi, L., and L. Galli. 2009. Interferon-gamma release assay improves the diagnosis of tuberculosis in children. Pediatric Infectious Disease Journal 28(6): 510-514.

27. Pottumarthy, S., A.J. Morris, A.C. Harrison, et al. 1999. Evaluation of the tuberculin $\gamma$ interferon assay: potential to replace the Mantoux skin test. Journal of Clinical Microbiology 37: 32293232.

28. Converse, P.J., S.L. Jones, and J. Astemborski. 1997. Comparison of a tuberculin interferon-gamma assay with the tuberculin skin test in high-risk adults: effect of human immunodeficiency virus infection. Journal of Infectious Diseases 176(1): 144-150.

29. Johnson, P.D.R., R.L. Stuart, and M.L. Grayson. 1999. Tuberculinpurified protein derivative-, MPT-64, ESAT-6-stimulated gamma interferon responses in medical students before and after Mycobacterium bovis BCG vaccination and in patients with tuberculosis. Clinical and Diagnostic Laboratory Immunology 6: 934-937.

30. Blussvan Oud-Alblas, H.J., M.E. van Vliet, and J.L. Kimpen. 2002. Human immunodeficiency virus infection in children hospitalized with tuberculosis. Annual Tropical Pediatric 22: $115-123$.

31. Taggart, E.W., H.R. Hill, R.G. Ruegner, et al. 2004. Evaluation of an in vitro assay for gamma interferon production in response to Mycobacterium tuberculosis infections. Clinical and DiagnosticLaboratory Immunology 11(6): 1089-1093. 